\title{
Picosecond Wireless Synchronization Using an Optically Locked Voltage Controlled Oscillator (OL-VCO)
}

\author{
Xuebei Yang, Xuyang Lu, and Aydin Babakhani \\ Department of Electrical and Computer Engineering, Rice University, Houston, TX, 77005, USA
}

\begin{abstract}
In this paper, the first fully integrated Optically Locked Voltage Controlled Oscillator (OL-VCO) is reported. The OL-VCO is locked to an RF source through a free-space optical link at $\lambda=850 \mathrm{~nm}$. In the locked mode, the OL-VCO achieves an RMS jitter of $1.6 p s e c$ with 16 averaging. It also improves the phase noise by $25 \mathrm{~dB}$ at $100 \mathrm{~Hz}$ offset frequency. The wireless synchronization is achieved at a distance of $1.5 \mathrm{~m}$, representing more than two orders of magnitude improvement compared to the prior art.
\end{abstract}

Index Terms - Wireless synchronization, precision timing, optical injection, voltage controlled oscillator, CMOS, SOI.

\section{INTRODUCTION}

In a distributed Multiple-Input-Multiple-Output (MIMO) array with widely spaced antennas, precision time synchronization among array elements is essential in achieving coherent combining of signals in space. In a conventional MIMO or phased array, the synchronization is achieved through wired links. Unfortunately, a wired link limits the flexibility of the array, increases the latency, and cannot be used where array elements are mobile. In this case, a wireless microwave signal may be used to synchronize the array elements. While wireless synchronization improves the flexibility and reconfigurability of an array, it suffers from the multi-path problem [1]. In a multi-path environment, both the amplitude and phase of the synchronization signal becomes noisy due to the time-varying electromagnetic scattering, which results in a large timing jitter. In order to minimize the multipath effects and reduce the timing jitter, an optical link can be used [2-4]. Since the optical beam has a very high directivity, the multi-path problem is alleviated and a much cleaner synchronization signal can be recovered. Additionally, due to the negligible coupling between the microwaves and optical waves, the timing jitter of the optical reference remains low. In the prior arts [2-4], discrete components and nonsilicon materials were used to establish a free-space optical synchronization. Unfortunately, these solutions are not compatible with CMOS-based integrated circuits and hence have niche applications.

In this work, for the first time, we report a fully integrated Optically-Locked Voltage Controlled Oscillator (OL-VCO) that operates based on the concept of spatial injection locking. The proposed OL-VCO is implemented using a commercial CMOS process technology and can be readily integrated with complex digital circuits. It achieves a picosecond timing jitter at a transmitter-receiver distance of larger than one meter. This represents more than two orders of magnitude improvement over the prior art [5].

\section{OL-VCO IMPLEMENTATION}

In Figure 1, the schematic of the OL-VCO is presented, which adopts a differential cross-coupled structure. The OLVCO can operate in both locked and free-running modes. In the locked mode, a modulated optical beam injects RF current by illuminating a custom photodiode integrated with the LC tank of the OL-VCO, as highlighted in Figure 1. In order to couple the optical beam from the front side of the chip, the metal fills above one of the photodiodes are blocked by using a fill-block layer. The other photodiode is covered by a large piece of metal to minimize optical injection. This asymmetric design maximizes the differential component of the injection current.

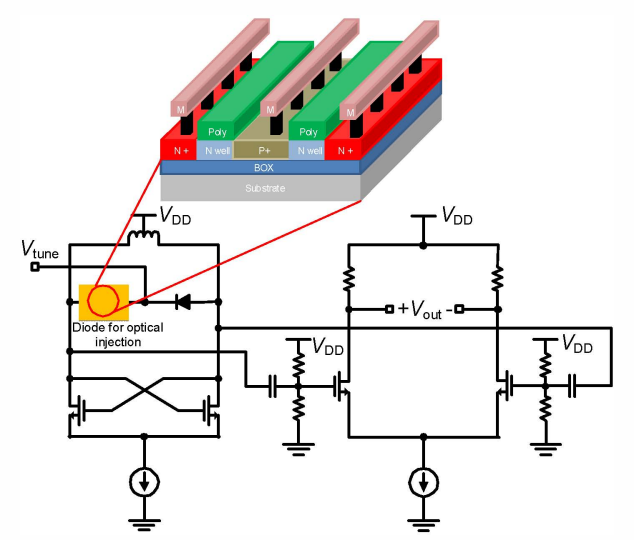

Figure 1 : Schematic of the OL-VCO with a differential buffer.

In an LC oscillator with a free-running frequency $\omega_{0}$, the oscillation frequency can be locked to $\omega_{\text {inj }}$ if a current with frequency $\omega_{\mathrm{inj}}$ is injected and $\omega_{\mathrm{inj}}$ satisfies

$$
\left|\omega_{0}-\omega_{i n j}\right| \leq \omega_{L}=\frac{\omega_{0}}{2 Q} \cdot \frac{I_{i n j}}{I_{o s c}}
$$

where $\mathrm{Q}$ is the quality factor of the LC tank. $\mathrm{I}_{\mathrm{inj}}$ and $\mathrm{I}_{\text {osc }}$ are the injection current and the transistor current, respectively. $\omega_{\mathrm{L}}$ is denoted as the locking range [6]. For a given oscillator with fixed $I_{o s c}, Q$ and $\omega_{0}$, the injection current $I_{\text {inj }}$ should be maximized to achieve a larger locking range. 
The implementation of high-speed photodiodes in a conventional CMOS process is a critical part of the design. In the CMOS process used in this work, no dedicated photodiodes are offered by the foundry. Therefore, photodiodes in this work are built using a standard $\mathrm{P}+/ \mathrm{N}-$ well diode offered by the foundry [7]. The polysilicon layer, shown in Figure 1, is used by the foundry for self-alignment purposes and does not impact the operation of the photodiode. By optimizing the layout of the $\mathrm{P}+/ \mathrm{N}$-well diode, a $50 \mu \mathrm{m}$ by $50 \mu \mathrm{m}$ photodiode achieves a $3 \mathrm{~dB}$ bandwidth of $1.8 \mathrm{GHz}$, as shown in Figure 2.

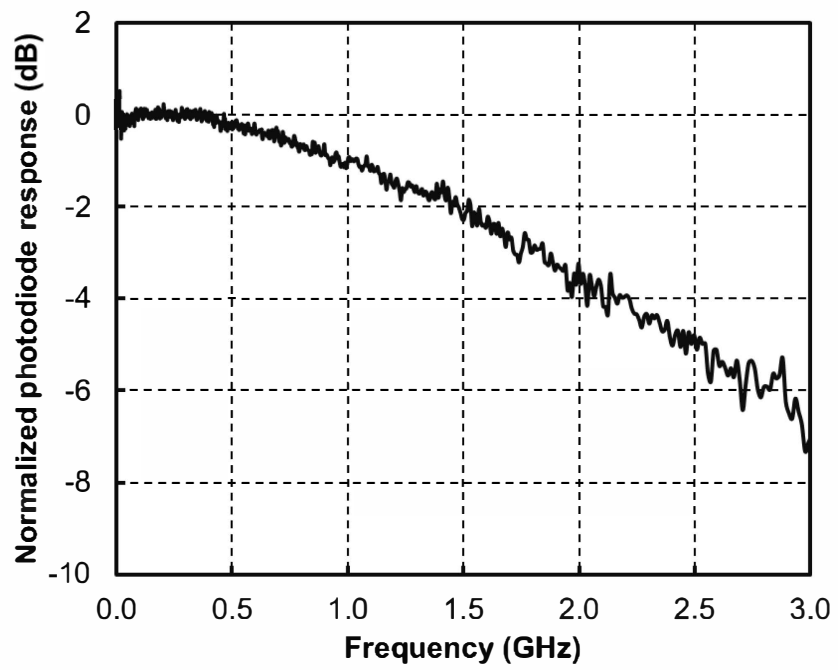

Figure 2: Normalized photodiode response versus frequency on a $50 \Omega$ load. The $3 \mathrm{~dB}$ bandwidth is approximately $1.8 \mathrm{GHz}$.

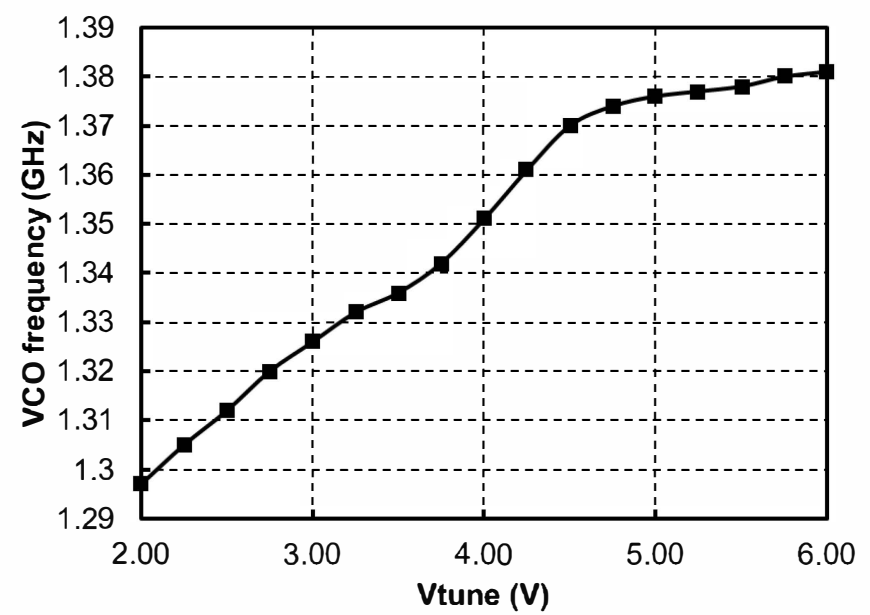

Figure 3: Oscillation frequency as a function of the tuning voltage in the free-running mode.

In addition to converting the optical beam to an electrical current, the photodiodes also behave as the varactors of the $\mathrm{OL}-\mathrm{VCO}$ to tune the free-running oscillation frequency. At a supply voltage of $1.3 \mathrm{~V}$, the free-running oscillation frequency of the OL-VCO can be tuned from $1295 \mathrm{MHz}$ to $1381 \mathrm{MHz}$, as shown in Figure 3. In order to deliver power to a $50 \Omega$ load, a differential buffer follows the OL-VCO. The power consumption of the OL-VCO and its buffer is $26 \mathrm{~mW}$.

\section{MEASUREMENT}

A block diagram of the measurement setup is presented in Figure 4. A Vertical Cavity Surface Emitting Laser (VCSEL) operating at $850 \mathrm{~nm}$ with $11 \mathrm{GHz}$ bandwidth is used as the optical source. The amplitude of the optical signal is modulated by an RF signal generator, Anritsu 68369B. The modulation frequency is close to the free-running frequency of the OL-VCO. The laser beam is collimated and focused onto the photodiode of the OL-VCO through two lenses. The distance between the VCSEL and the OL-VCO chip is approximately 1.5 meters.

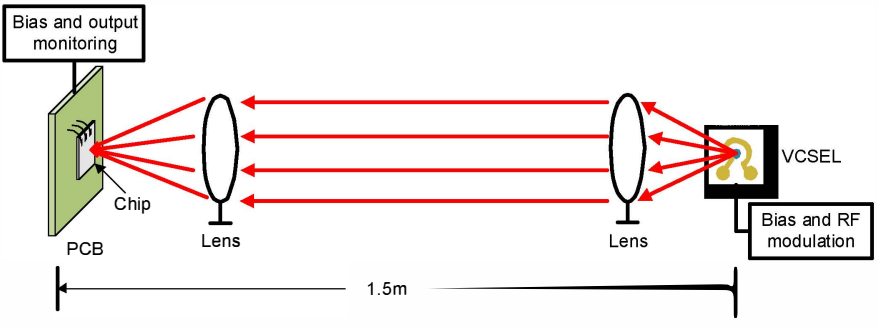

Figure 4 : Block diagram of the measurement setup.

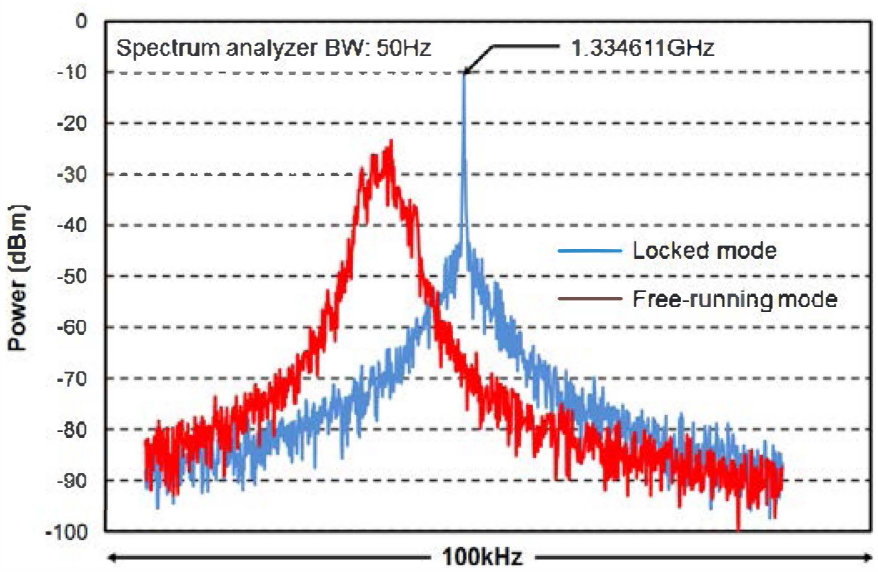

Figure 5: Measured power spectrum of the OL-VCO in both freerunning and locked modes.

Figure 5 presents the frequency spectrum of OL-VCO in both free-running and locked modes. The measurement is done using an Agilent N9030A signal analyzer. The supply and tuning voltages are kept constant at $1.3 \mathrm{~V}$ and $5.5 \mathrm{~V}$, respectively. The RF power of the laser beam is $-3 \mathrm{dBm}$. Based on results shown in Figure 5, it is concluded that the OL-VCO in the locked mode generates a sharp tone at the injection frequency. Figure 6 shows the measured phase-noise of the $\mathrm{OL}-\mathrm{VCO}$ in both free-running and locked modes. The measured phase-noise of the OL-VCO in the free-running mode at $100 \mathrm{~Hz}$ and $1 \mathrm{kHz}$ offset from the carrier frequency is $32 \mathrm{dBc} / \mathrm{Hz}$ and $-40.3 \mathrm{dBc} / \mathrm{Hz}$, respectively. These values improve to $-57 \mathrm{dBc} / \mathrm{Hz}$ and $-66.5 \mathrm{dBc} / \mathrm{Hz}$ in the locked mode. Based on these measured values, the phase noise improves by about $25 \mathrm{~dB}$. The calculated RMS jitter by integrating the phase noise from $100 \mathrm{~Hz}$ to $1 \mathrm{MHz}$ is $81 \mathrm{psec}$ and $3.1 \mathrm{psec}$ for the OL-VCO in the free-running and locked modes, 
respectively. An Agilent DCA-X 86100D sampling oscilloscope is also used to measure the timing jitter of the OL-VCO in the locked mode. The measured RMS jitter is $1.6 \mathrm{psec}$ with 16 averaging, as shown in Figure 7 . Both the phase-noise and timing jitter of the OL-VCO in the locked mode are limited by the phase-noise of the RF signal generator (Anritsu 68369B), which is used to modulate the VCSEL. It is possible to further improve the phase noise and jitter by using a more stable RF source. In order to verify that the locking is not caused by the RF radiation leakage, the laser beam is blocked and it is confirmed that no locking can be achieved afterwards. To the best knowledge of the authors, this is the first demonstration of wireless synchronization with picosecond timing jitter at a distance larger than 1 meter using a CMOS platform. It extends the $2.5 \mathrm{~mm}$ distance achieved previously [5] by more than two orders of magnitude.

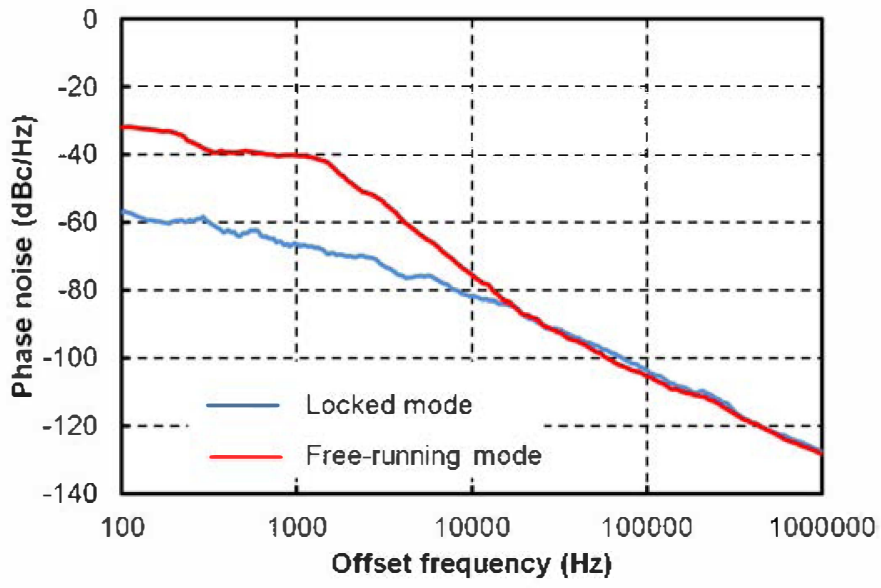

Figure 6 : Measured phase noise of the OL-VCO in both freerunning and locked modes.

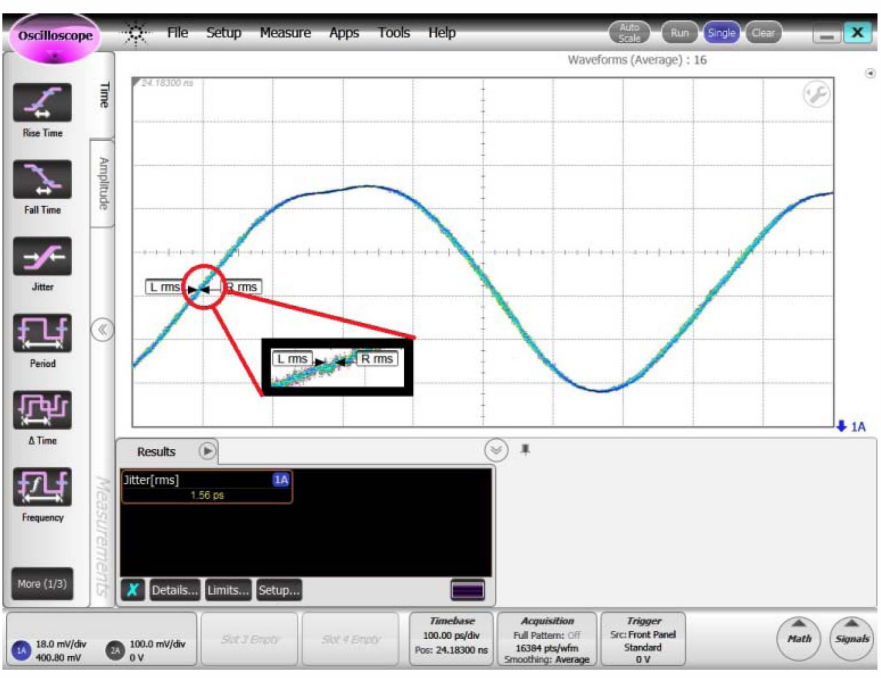

Figure 7 : Timing jitter of the OL-VCO in the locked mode.

To complete the measurements, the locking range of the OL-VCO in the locked mode is investigated. As the locking range is proportional to the injection current, the laser power directly influences the locking range. Another factor that impacts the locking range is the reverse bias voltage of the photodiode, which is the difference between the voltage of the tuning and supply nodes. As the reverse bias increases, the optoelectronic conversion efficiency also increases. Therefore, for a given laser power, a larger injection current can be generated. The measured locking ranges for tuning voltages of $3 \mathrm{~V}, 4 \mathrm{~V}$ and $5.5 \mathrm{~V}$, corresponding to reverse bias voltages of $1.7 \mathrm{~V}, 2.7 \mathrm{~V}$, and $4.2 \mathrm{~V}$, are shown in Figure 8 . It is confirmed that the locking range decreases as the laser power drops. For a tuning voltage of $5.5 \mathrm{~V}$, the OL-VCO can be locked with a laser power as low as $-18 \mathrm{dBm}$.

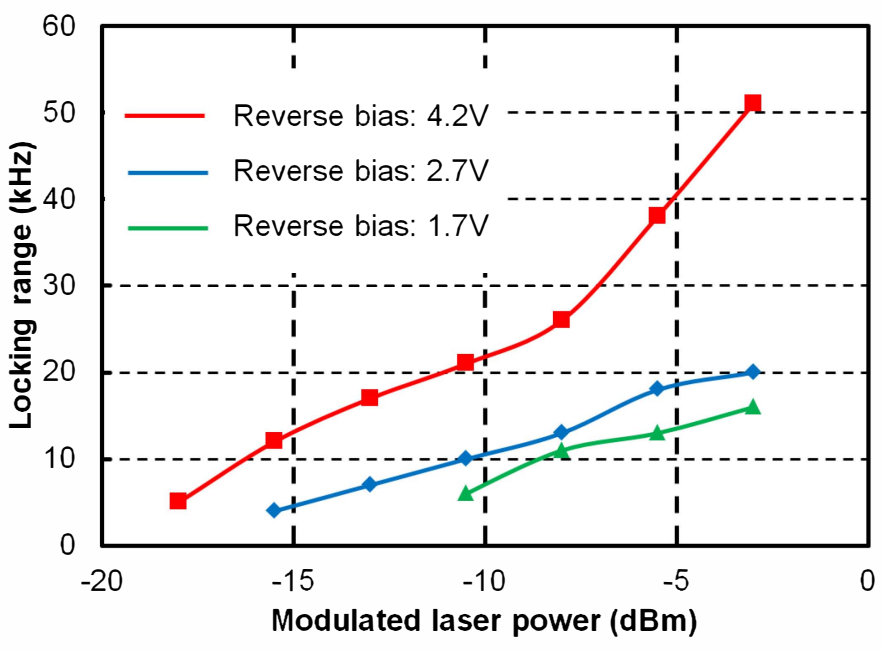

Figure 8 : Locking range of the OL-VCO as a function of the laser power and the bias of the photodiode.

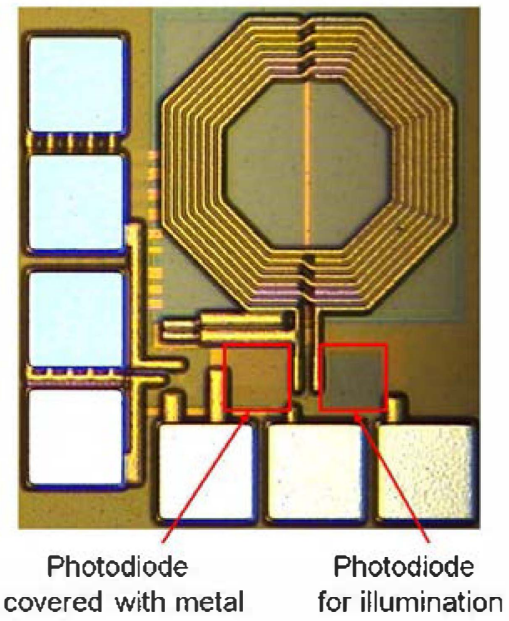

Figure 9: Micrograph of the chip.

Finally, we show the micrograph of the chip in Figure 9. The chip occupies an area of $550 \mu \mathrm{m}$ by $500 \mu \mathrm{m}$, including the pads. This chip is fabricated in IBM $0.18 \mu \mathrm{m}$ CMOS SOI process technology without any process-tuning or postprocessing. 


\section{CONCLUSION}

In this paper, we have presented, for the first time, a fullyintegrated Optically-Locked VCO (OL-VCO). At a distance of $1.5 \mathrm{~m}$, the proposed OL-VCO is synchronized to an RF source with picosecond timing jitter via a free-space optical link. This represents more than two orders of magnitude improvement in distance compared to prior art implemented in CMOS [5].

\section{REFERENCES}

[1] W. Jakes, Microwave Mobile Communications, Wiley, 1994.

[2] A. Seeds, A. A. A. de Salles, "Optical Control of Microwave Semiconductor Devices", IEEE Trans. Microwave Theory \& Tech., vol. 38, no. 5, pp. 577-584, 1990.

[3] A. A. A. de Salles, "Optical control of GaAs MESFETs," IEEE Trans. Microwave Theory \& Tech., vol. 31, no. 10, pp. 812 820, 1983.

[4] A. A. de Salles and J. R. Forrest, "Initial observations of optical injection locking of GaAs metal semiconductor field effect transistor oscillators," Appl. Phys. Lett., vol. 38, no. 5, pp. 392394, Mar. 1981.

[5] X. Guo, et al., "A Receiver with Start-up Initialization and Programmable Delays for Wireless Clock Distribution", ISSCC Dig. Tech. papers, pp. 386-387, Feb. 2006.

[6] B. Razavi, "A study of injection locking and pulling in oscillators", IEEE J. Solid-State Circuits, vol. 39, pp. 14151424, 2004.

[7] X. Yang, X. Lu, A. Babakhani, "Impact of Layout on the Performance of Photodiodes in $0.18 \mu \mathrm{m}$ CMOS SOI", IEEE Photonics Conference, ThA1.3, 2013. 